\title{
Does screening for congenital cytomegalovirus at birth improve longer term hearing outcomes?
}

\author{
Cathie Hilditch, ${ }^{1,2}$ Bianca Liersch, ${ }^{3}$ Nicola Spurrier, ${ }^{4,5}$ Emily J Callander, ${ }^{6}$ Celia Cooper, \\ Amy K Keir ${ }^{1,2,8}$
}

\begin{abstract}
- Additional material is published online only. To view please visit the journal online (http://dx.doi.org/10.1136/ archdischild-2017-314404).
\end{abstract}

${ }^{1}$ Healthy Mothers, Babies and Children Theme, South Australian Health and Medical Research Institute, North Adelaide, South Australia, Australia

${ }^{2}$ Robinson Research Institute and the Adelaide Medical School, University of Adelaide, North Adelaide, South Australia, Australia

${ }^{3}$ Children's Audiology Service, Women's and Children's Health Network, North Adelaide, South Australia, Australia

${ }^{4}$ South Australian Department

of Health and Ageing, Adelaide,

South Australia, Australia

${ }^{5}$ Department of Paediatrics and Child Health, Flinders University of South Australia, Adelaide,

South Australia, Australia

${ }^{6}$ Australian Institute of Tropical Health and Medicin, James Cook University, Townsville, Queensland, Australia Department of Infectious Diseases, Women's and Children's Hospital, North Adelaide, South Australia, Australia

${ }^{8}$ Department of Neonatal Medicine, Women's and Children's Hospital, North Adelaide, South Australia, Australia

\section{Correspondence to} Dr Amy K Keir, Department of Neonatal Medicine, Women's and Children's Hospital, North Adelaide, SA, 5006: amy.keir@adelaide.edu.au

Received 31 October 2017 Revised 11 April 2018 Accepted 12 April 2018 Published Online First 28 April 2018

Check for updates

To cite: Hilditch C, Liersch B, Spurrier $\mathrm{N}$, et al.

Arch Dis Child

2018;103:988-992.

\section{ABSTRACT}

Currently, the diagnosis of congenital cytomegalovirus (cCMV) infection in most highly resourced countries is based on clinical suspicion alone. This means only a small proportion of CCMV infections are diagnosed. Identification, through either universal or targeted screening of asymptomatic newborns with $\mathrm{CCMV}$, who would previously have gone undiagnosed, would allow for potential early treatment with antiviral therapy, ongoing audiological surveillance and early intervention if sensorineural hearing loss (SNHL) is identified. This paper systematically reviews published papers examining the potential benefits of targeted and universal screening for newborn infants with cCMV. We found that the treatment of these infants with antiviral therapy remains controversial, and clinical trials are currently underway to provide further answers. The potential benefit of earlier identification and intervention (eg, amplification and speech therapy) of children at risk of later-onset SNHL identified through universal screening is, however, clearer.

\section{INTRODUCTION}

Congenital cytomegalovirus (cCMV) is the most common intrauterine infection in highly resourced countries, occurring in $0.4 \%-0.64 \%$ of all births. ${ }^{12}$ The true incidence of the disease is likely to be higher, as up to $90 \%$ of infected infants are asymptomatic at birth. Up to $10 \%-25 \%$ of all childhood sensorineural hearing loss (SNHL) is attributed to CCMV infection, making cytomegalovirus (CMV) the largest non-genetic cause of SNHL in children. ${ }^{3}$ cCMV-related SNHL is progressive in more than $50 \%$ of cases. ${ }^{1}$ Identification, through either universal or targeted screening of asymptomatic newborns with cCMV, who would previously have gone undiagnosed, would allow for potential early treatment with antiviral therapy, ongoing audiological surveillance and early intervention if SNHL is identified. Early identification of hearing loss is associated with improved language development. ${ }^{4}$ Antiviral therapy has been shown to reduce hearing deterioration in symptomatic children with cCMV if started within the first month of birth. ${ }^{5}$

Our review will address the structured clinical question 'In asymptomatic newborn infants in highly resourced countries (P), does screening for cCMV using saliva PCR testing (I) compared to not screening $(\mathrm{C})$ allow for earlier identification of infants with CMV-related SNHL and effective early intervention/treatment?'. It will also assess cCMV screening against the WHO screening criteria.
What is already known on this topic?

- Congenital cytomegalovirus (cCMV) is the largest non-genetic cause of sensorineural hearing loss (SNHL) in children.

- cCMV-related SNHL is progressive in more than $50 \%$ of cases, and affected infants are likely to pass their newborn hearing screen.

\section{What this study adds?}

- Identification, through either universal or targeted screening of asymptomatic newborns with $\mathrm{CCMV}$, who would previously have gone undiagnosed.

- This would allow for potential early treatment with antiviral therapy if other symptoms are identified, ongoing audiological surveillance and early intervention if SNHL develops.

\section{Definitions}

The definition of symptomatic cCMV infection varies in the literature. ${ }^{6}$ For the purposes of this review, children are classified as symptomatic if they have acute signs present at birth. These signs include petechiae, jaundice, hepatosplenomegaly, microcephaly (head circumference $<2$ nd centile or 2 SD below the mean), SNHL, small for gestational age (birth weight $<10$ th centile), chorioretinitis and seizures. Targeted screening is defined as screening limited to those children having failed their newborn hearing screen.

\section{Search strategy}

MEDLINE via Ovid (1946 to 3 June 2017), Embase via Ovid (1974 to 11 July 2017) and the Maternity and Infant Care Database (1971 to June 2017) were searched for relevant articles. Full search strategies and detailed results are availablein the online supplementary data. A total of 124 articles, after removal of duplicates, were found and screened by title and abstract. Twenty-nine full-text articles were subsequently reviewed, and six articles were included as relevant to the clinical question. These are summarised in tables 1 and 2 .

\section{Newborn screening for cCMV infection}

To allow for better understanding of whether cCMV may be an appropriate condition to screen 
Table 1 Universal screening

\begin{tabular}{|c|c|c|c|c|c|}
\hline Citation, country & Study group & $\begin{array}{l}\text { Study type (level of } \\
\text { evidence)* }\end{array}$ & Outcome & Key result & Comments \\
\hline
\end{tabular}

CMV, cytomegalovirus; CCMW, congenital cytomegalovirus; SNHL, sensorineural hearing loss.

for in all newborn infants, it will be assessed against the WHO criteria (box 1):

\section{Important health problem}

cCMV is recognised as the most common preventable infective cause of SNHL in children and is the leading non-genetic cause of SNHL. It is a significant public health problem. ${ }^{78}$

\section{Accepted treatment or intervention for patients with recognised disease: antiviral therapy}

Treatment of newborns with symptomatic cCMV with antiviral therapy has been shown to improve hearing and developmental outcomes. There is evidence that the use of 6 weeks of ganciclovir improves or maintains hearing outcomes at 6 months if started within the first month of life. ${ }^{5}$ A subsequent study demonstrated that 6 months of valganciclovir delivers more sustained improvements in hearing and improves neurocognitive outcomes in newborns with cCMV. ${ }^{9}$

In terms of evidence for treatment of infants with isolated SNHL and confirmed CMV and no other symptoms, there have been no clinical trials to date and expert opinion varies. Many commentators recommend treatment, extrapolating from improved hearing outcomes in treated symptomatic infants. ${ }^{7}$ For a newborn with asymptomatic infection who passes the hearing screen, there is currently no data to support treatment. Similarly, there is no data to support treatment of CMV-related hearing loss presenting or developing beyond the first month of life. All of these patient groups are currently the subject of ongoing clinical trials. ${ }^{8}$

\section{Accepted treatment or intervention for patients with recognised disease: audiological surveillance}

In approximately 50\% of children with SNHL due to cCMV, hearing loss will be progressive. ${ }^{7}$ Many of these children will be asymptomatic at birth and pass their newborn hearing screen. Early detection of subsequent hearing impairment in this group of children is one of the substantial benefits of universal screening for cCMV. This would allow augmentation of hearing and support for speech and language development at a much earlier age than currently possible. ${ }^{4}$

Facilities for diagnosis, treatment and follow-up should be available A cCMV screening programme may best be incorporated into existing newborn hearing or metabolic screening programme infrastructures. Confirmation of the diagnosis of cCMV would need to be made and infants then assessed by a paediatrician or infectious diseases physician. Ongoing follow-up of symptomatic infants with cCMV is currently the responsibility of general paediatricians, infectious disease physicians, audiologists and allied health professionals.

Asymptomatic newborns, identified solely through a universal cCMV screening programme, could either have normal or impaired hearing. The latter group would require paediatric, ear-nose-throat (ENT) surgical and audiological follow-up as currently provided for infants with SNHL. In addition, they would require closer monitoring of other aspects of development during early childhood because of the increased risk of subtle neurocognitive impairments associated with cCMV.

Asymptomatic newborns with normal hearing at birth but positive for CMV would also require initial medical review and increased hearing surveillance. ${ }^{7}$ Approximately 50\% of children with SNHL due to cCMV will experience progressive hearing loss. ${ }^{7}$ All children with cCMV should have their hearing tested regularly in early childhood to ensure the early detection of progressing SNHL. Specific recommendations for audiological monitoring are at least every 6 months until age 3 years and then yearly until 6 years old. ${ }^{7}$

Recognisable latent or early symptomatic stage and natural history Ten per cent of children with cCMV are symptomatic at birth, with characteristic sequelae of petechiae, jaundice, hepatosplenomegaly, microcephaly (head circumference $<2$ nd centile or 2 SD below the mean), SNHL, chorioretinitis and seizures. The remaining $90 \%$ of infants with cCMV will be asymptomatic, and $10 \%-15 \%$ of these children will develop SNHL over the first 5-7 years of life. For asymptomatic infants, this is their biggest future risk. As this hearing loss often has delayed onset, only $50 \%$ of hearing loss cases are detected in the neonatal period. $^{7}$ A recent systematic review found the cumulative risk of SNHL in children with asymptomatic cCMV with follow-up until 5 years was $7 \%-11 \% .^{10}$

Availability and acceptability of a suitable test

A highly specific and sensitive screening test is available for cCMV. CMV PCR ${ }^{11}$ using salivary sample collection is more convenient than urine collection, and CMV viral loads are highest in saliva. Sensitivity is reported at $97.4 \%-100 \%$ and specificity at 99.9 compared with rapid culture. ${ }^{2}$ High volume testing using 
- The condition sought should be an important health problem.

- There should be an accepted treatment for patients with recognised disease.

- Facilities for diagnosis and treatment should be available.

- There should be a recognisable latent or early symptomatic stage.

- There should be a suitable test or examination.

- The test should be acceptable to the population.

- The natural history of the condition, including development from latent to declared disease, should be adequately understood.

- There should be an agreed policy on whom to treat as patients.

- The cost of case-finding (including diagnosis and treatment of patients diagnosed) should be economically balanced in relation to possible expenditure on medical care as a whole.

- Case-finding should be a continuing process and not a 'once and for all' project.

commercial or inhouse assays is feasible. Australian estimates are that salivary PCR test will cost $\$ A 25.00$. Confirmatory testing will be required to confirm the diagnosis and eliminate false positives. Shedding of the CMV virus occurs for long periods of time, so there is an adequate window of time for confirmatory testing. However, if treatment with antiviral agents is being considered, then testing and confirmation of diagnosis would need to occur within 30 days of birth (see treatment below).

In the UK, salivary screening for CCMV within the newborn hearing screening programme was found to feasible, acceptable and identified infants with cCMV-related SNHL. ${ }^{12}$ Only infants who failed their initial newborn hearing screen were tested, and feasibility was defined as the ability to take samples before 3 weeks of age and clinical assessment by 30 days of age. Maternal anxiety levels at referral and 3 months later were not increased. ${ }^{12}$ The study reported that $98 \%$ of the study population successfully provided a salivary swab within the 3 -week period. Kadambari et al $^{13}$ included an assessment of the feasibility of introducing targeted screening within the UK, and all healthcare professional involved agreed it was feasible to introduce a targeted screening programme. The acceptability of either targeted or universal screening for CMV to families would need to be addressed during the development of potential programmes.

All individual studies included in this review (tables 1 and 2) used salivary PCR as the screening tool. All demonstrated that introducing either universal or targeted screening programmes was feasible.

\section{Agreed policy on whom to treat as patients: antiviral therapy}

This area remains controversial with respect to antiviral treatment. Many recommendations are based on varying expert opinions. Our recommendations, on review of the evidence-base, for which infants would benefit from antiviral treatment would be:

- In symptomatic newborns ( $<30$ days of age):

- If child is severely unwell, initial treatment with intravenous ganciclovir (not for $>6$ weeks). Treatment should then be continued with oral valganciclovir to complete a total course (intravenous/oral combined) of 6 months.

- If child is well/mildly unwell and able to tolerate oral therapy, treatment with oral valganciclovir for 6 months 
has been shown to have better hearing and developmental outcome at 12 and 24 months of age. ${ }^{9}$

- Asymptomatic newborns (<30 days of age): no treatment.

- Asymptomatic newborns with SNHL (<30 days of age): no currently available data to support treatment; some experts consider treatment up to 6 months with oral valganciclovir. ${ }^{14}$

- Children with CMV-related SNHL identified or developing beyond the neonatal period: no currently available data to support treatment.

Agreed policy on whom to treat as patients: increased audiological surveillance

The potential positive impact on hearing outcomes through implementation of increased surveillance of asymptomatic newborn identified with cCMV through a screening programme needs to be considered.

\section{Cost-effectiveness of targeted and universal screening}

A recently published study has assessed the cost-effectiveness of implementing targeted or universal screening for CCMV, relative to no screening in the USA. ${ }^{15}$ Costs included the direct costs to the healthcare system, and a number of outcomes were assessed. The study estimated an incremental cost of US\$10 000 per case of cCMV infection detected, US\$90 039 per case cCMV hearing loss detected and US\$12 620277 per cochlear implant avoided for universal screening, and US\$2832 per case of cCMV infection detected, US\$3 916 per case cCMV hearing loss detected and US\$271 947 per cochlear implant avoided for targeted screening.

A study in the UK estimated the additional cost of 'protecting' hearing in one infant using a targeting screening programme. Based on a valganciclovir treatment efficacy rate of $47 \%$, the study estimated it would cost $£ 14202$ to improve the hearing of one infant. ${ }^{16}$ In order to provide more comparable results, future cost-effectiveness studies should estimate quality-adjusted life year outcomes associated with different treatment options. This would be in align with reporting recommendations of medical service appraisal committees internationally. ${ }^{17} 18$

\section{DISCUSSION}

Currently, diagnosis of cCMV infection in most highly resourced countries is based on clinical suspicion, meaning only a small proportion of cCMV infections are diagnosed.

\section{Universal screening}

Universal newborn screening would aim to identify asymptomatic infected infants for ongoing monitoring for delayed-onset hearing loss and appropriate follow-up. This hearing loss will not be detected by existing newborn hearing screening protocols. Table 1 outlines two prospective cohort studies examining universal screening for cCMV and follow-up with hearing screening. Leruez-Ville et $a l^{19}$ identified 4 out of 51 newborns with cCMV who had hearing impairment detected on further audiological assessment. This study raised the possibility of detecting false positives when using salivary PCR for screening through postbirth contamination (CMV being often present in breast milk and in the birth canal). Eighty-seven infants in the study were detected as positive for cCMV on their first screen, but on repeat salivary PCR screening, only 51 newborns were confirmed as being truly positive for cCMV. Barkai et al ${ }^{20}$ conducted a universal screening programme and found 2 of 47 infants with hearing loss, one of these being late onset. All infants had normal initial hearing screening results; however, infants with cCMV were followed up with later audiological assessments.

\section{Targeted screening}

Table 2 includes four studies looking at targeted screening for cCMV. All of these studies screened infants who failed their initial newborn hearing screen. One prospective cohort study ${ }^{21}$ identified six infants with cCMV and SNHL. All infants were treated with valganciclovir for 6 weeks, with only one child experiencing an improvement in hearing at 1 year of age. Williams et $a l^{12}$ included an assessment of the feasibility and acceptability of targeted screening and found no increase in maternal anxiety in infants screened for cCMV. They reported that $98 \%$ of the study population provided a salivary swab within the 3 -week period. Kadambari et $a l^{13}$ included an assessment of the feasibility of introducing targeted screening within the UK, and all healthcare professional involved agreed it was feasible to introduce a screening programme. Diener et $a l^{22}$ evaluated the success of a targeted cCMV screening programme and found that with the introduction of targeted cCMV screening, timely diagnostic hearing evaluation rates improved, from $56 \%$ to $77 \%$. The four studies evaluating a targeted screening approach acknowledged the limitations of targeted screening missing those infants who pass their initial hearing test. The main reason for not advocating for universal screening was limited knowledge about the feasibility and cost-effectiveness of such a screening strategy.

To summarise, screening for cCMV infection could result in: (1) increased recognition of mildly symptomatic infants (universal screening), (2) diagnosis of cCMV in infants with known SNHL (targeted screening) and (3) increased detection of asymptomatic infants at future increased risk of SNHL (universal screening). In group one, there is sufficient evidence to support the treatment of mildly symptomatic infants. ${ }^{59}$ In group two, it is clear that infants with SNHL will significantly benefit from audiological amplification and appropriate speech and language intervention. However, there is no clear evidence that antiviral therapy will improve outcomes for infants with isolated SNHL secondary to cCMV. Further research is needed to answer this question and this is underway. ${ }^{8}$ For group three, the length of time and frequency of audiological follow-up of cCMV positive asymptomatic infants without SNHL would require consideration. Current recommendations are up until 6 years of age. ${ }^{7}$

Universal screening would identify all three groups previously outlined. Targeted screening would lead to identification of infants in group two and potentially some infants with other symptoms in addition to SNHL.

\section{CONCLUSIONS}

In answering our research question 'In asymptomatic newborn infants in highly resourced countries (P), does screening for cCMV using saliva PCR testing (I) compared to not screening (C) allow for earlier identification of infants with CMV-related SNHL and effective early intervention/treatment?', we conclude that universal screening would allow for earlier identification of infants with at risk of CMV-related isolated SNHL. Unfortunately, the treatment of these infants with antiviral therapy remains controversial, and clinical studies are currently underway that will hopefully provide evidence of treatment efficacy. Other benefits (early intervention and hearing augmentation) of early identification of hearing impairment in these children at risk of later-onset SNHL, are well documented. ${ }^{4}$ 
Acknowledgements We would like to thank Natalie Dempster, Librarian at the Women's and Children's Hospital, Adelaide, Australia, for her invaluable assistance with the search strategies.

Contributors AKK had the idea for the article. $\mathrm{CH}$ performed the literature search. All authors wrote the paper together. AKK is the guarantor for the paper.

Funding The authors have not declared a specific grant for this research from any funding agency in the public, commercial or not-for-profit sectors.

Competing interests None declared.

Provenance and peer review Not commissioned; externally peer reviewed.

\section{Data sharing statement Not applicable.}

(C) Article author(s) (or their employer(s) unless otherwise stated in the text of the article) 2018. All rights reserved. No commercial use is permitted unless otherwise expressly granted.

\section{REFERENCES}

1 Kenneson A, Cannon MJ. Review and meta-analysis of the epidemiology of congenital cytomegalovirus (CMV) infection. Rev Med Virol 2007;17:253-76.

2 Boppana SB, Ross SA, Shimamura M, et al. Saliva polymerase-chain-reaction assay for cytomegalovirus screening in newborns. N Engl J Med 2011;364:2111-8.

3 Grosse SD, Ross DS, Dollard SC. Congenital cytomegalovirus (CMV) infection as a cause of permanent bilateral hearing loss: a quantitative assessment. J Clin Virol 2008;41:57-62.

4 Ching TY. Is Early Intervention Effective in Improving Spoken Language Outcomes of Children With Congenital Hearing Loss? Am J Audiol 2015;24:345-8.

5 Kimberlin DW, Lin CY, Sánchez PJ, et al. Effect of ganciclovir therapy on hearing in symptomatic congenital cytomegalovirus disease involving the central nervous system: a randomized, controlled trial. J Pediatr 2003;143:16-25.

6 Cannon MJ, Griffiths PD, Aston V, et al. Universal newborn screening for congenital CMV infection: what is the evidence of potential benefit? Rev Med Virol 2014;24:291-307.

7 Kadambari S, Williams EJ, Luck S, et al. Evidence based management guidelines for the detection and treatment of congenital CMV. Early Hum Dev 2011;87:723-8.

8 Marsico C, Kimberlin DW. Congenital Cytomegalovirus infection: advances and challenges in diagnosis, prevention and treatment. Ital J Pediatr 2017;43:38.

9 Kimberlin DW, Jester PM, Sánchez PJ, et al. Valganciclovir for symptomatic congenital cytomegalovirus disease. N Engl J Med 2015;372:933-43.
10 Bartlett AW, McMullan B, Rawlinson WD, et al. Hearing and neurodevelopmental outcomes for children with asymptomatic congenital cytomegalovirus infection: a systematic review. Rev Med Virol 2017:e1938.

11 Ross SA, Ahmed A, Palmer AL, et al. Detection of congenital cytomegalovirus infection by real-time polymerase chain reaction analysis of saliva or urine specimens. J Infect Dis 2014;210:1415-8.

12 Williams EJ, Kadambari S, Berrington JE, et al. Feasibility and acceptability of targeted screening for congenital CMV-related hearing loss. Arch Dis Child Fetal Neonatal Ed 2014;99:F230-6.

13 Kadambari S, Luck S, Davis A, et al. Evaluating the feasibility of integrating salivary testing for congenital CMV into the Newborn Hearing Screening Programme in the UK. Eur J Pediatr 2015;174:1117-21.

14 Luck SE, Wieringa JW, Blázquez-Gamero D, et al. Congenital Cytomegalovirus: a European expert consensus statement on diagnosis and management. Pediatr Infect Dis J 2017;36:1205-13.

15 Gantt S, Dionne F, Kozak FK, et al. Cost-effectiveness of Universal and Targeted Newborn Screening for Congenital Cytomegalovirus Infection. JAMA Pediatr 2016;170:1173-80.

16 Williams EJ, Gray J, Luck S, et al. First estimates of the potential cost and cost saving of protecting childhood hearing from damage caused by congenital CMV infection. Arch Dis Child Fetal Neonatal Ed 2015;100:F501-6.

17 National Institute for Health and Care Excellence (NICE). Guide to the Methods of Technology Appraisal. London, UK: NICE, 2013.

18 Medical Services Advisory Committee (MSAC). Technical Guidelines for preparing assessment reports for the Medical Services Advisory Committee (Version 3.0. Canberra, Australia: Medical Services Advisory Committee (MSAC), 2017.

19 Leruez-Ville M, Magny JF, Couderc S, et al. Risk factors for congenital cytomegalovirus infection following primary and nonprimary maternal infection: a prospective neonatal screening study using polymerase chain reaction in Saliva. Clin Infect Dis 2017;65:398-404.

20 Barkai G, Ari-Even Roth D, Barzilai A, et al. Universal neonatal cytomegalovirus screening using saliva - report of clinical experience. J Clin Virol 2014;60:361-6.

21 Kawada J, Torii Y, Kawano Y, et al. Viral load in children with congenital cytomegalovirus infection identified on newborn hearing screening. J Clin Virol 2015;65:41-5.

22 Diener ML, Zick CD, McVicar SB, et al. Outcomes From a Hearing-Targeted Cytomegalovirus Screening Program. Pediatrics 2017;139:e20160789.

23 Wilson JM, Jungner YG. [Principles and practice of mass screening for disease]. Bol Oficina Sanit Panam 1968;65:281-393. 